\title{
LOW COST MICROSCOPY FOR THREE DIMENSIONAL IMAGING USING DIGITAL INLINE HOLOGRAPHIC PRINCIPLE
}

\author{
G.Hanu Phani Ram¹, Vimal Prabhu Pandiyan², Renu John ${ }^{3}$ \\ ${ }^{1}$ Research Scholar, Biomedical Engineering, IIT Hyderabad, Telangana, India \\ ${ }^{2}$ Research Scholar, Biomedical Engineering, IIT Hyderabad, Telangana, India \\ ${ }^{3}$ Associate professor and Head of department, Biomedical Engineering, IIT Hyderabad, Telangana, India
}

\begin{abstract}
Optical microscopy is reached a new level in terms of resolution, 3-D imaging capability, flexibility of imaging different samples which increase imaging complexity and the cost. Though established labs can afford high-end microscopes, it remains a concern in rural areas where clinics and patients cannot afford much. Semi-portable microscopy based on inline holographic setup is demonstrated where depth information as 3rd dimension can also be accessed. This setup contains only light emitting diode (LED), pinhole and charge coupled device (CCD) camera. Since laser source gives rise to speckle noise and it is also cost constraint for developing a low cost microscopy, thus it is replaced with incoherent LED source. This setup is also known as 'lensless holography' because there is no use of lens for imaging. In conventional inline holographic setup the sample is placed closed to the pinhole which will restrict field of view (FOV) and diffraction signature of one particle (cell) will overlap with other. To avoid overlap of diffraction signatures and to increase FOV sample was placed close to CCD sensor. To test the working of microscopy agarose microbeads were used. Optimization algorithm is used for reconstruction of object field from recorded hologram. Thus both amplitude as well as the phase images of the microbeads is reconstructed. Instead of using microscopic objective to focus sample, autofocus algorithm is used to calculate the focused plane.
\end{abstract}

Keywords: lensless microscopy, LED, low cost

\section{INTRODUCTION}

Gabor invented a new microscopic technique [1]. The technique which he developed is known as in-line holography as both object wave and reference wave have common path. The object beam is diffracted part of light by object and reference beam is non diffracted part. These both will interfere at hologram plane where 'inline hologram' is recorded. Since both beams propagate in same direction, reconstructed object will be overlapped with twin image and reference intensity. In order to get rid of overlap between real, virtual and background intensity Leith and Upatnieks [2] came up with off-axis holographic setup which is still used as standard holographic technique for various applications. Formation of off-axis holography is similar to the Gabor's in-line holography. It is recorded by the interference of object beam and reference beam. Object beam is the wave field that is scattered from the object whereas the reference beam is the source wave field. Advantage of off-axis holography is that it can separate the real and virtual images spatially as the direction of propagation of object and object beam are separated by an angle $\theta$. Off-axis setup can be used for reconstruction of object as it can easily filter out virtual and background [3, 4]. But inline holography is attractive in terms of its simple setup, high resolution of thick volume specimens and insensitive to the instabilities. The main drawback is to eliminate twin image. Gabor proposed that the twin image problem can be eliminated with two holograms of the object which are reconstructed using quadrature reference waves. Koren et.al [5] proposed an iterative algorithm based on finite support constraint for twin image elimination. This method works only when hologram is dominated by holographic diffraction terms. Some twin image elimination methods don't require any object support where twin image is eliminated by constraints [6]. In present work Constraint optimization problem [7] was used for recovery of object by elimination of twin image and zeroth order term. Most of the holographic technique uses laser as a source as it has coherent property, one disadvantage of using laser source is that it gives speckle noise. It can be replaced it with low cost LED source. This setup would also avoid small aperture pinholes around $1 \mu \mathrm{m}$ size [8] as they require stable and precise setup to drive light through such pinholes. The reconstruction of the object recorded digitally by charge coupled device (CCD) is known as the 'Digital holography'. The whole optical wave field of the object can be reconstructed by mathematical modeling of the light propagation using the properties like interference and diffraction which are the basis for holography.

In this work a low cost imaging device for rural health care is demonstrated. Thus setup consists of LED, pinhole and camera for imaging microbeads.

\section{OPTICAL SETUP}

The optical setup for inline holography is given in the fig 1 . It is simple setup consists of LED source, pinhole $(50 \mu \mathrm{m})$ and CCD detector. 


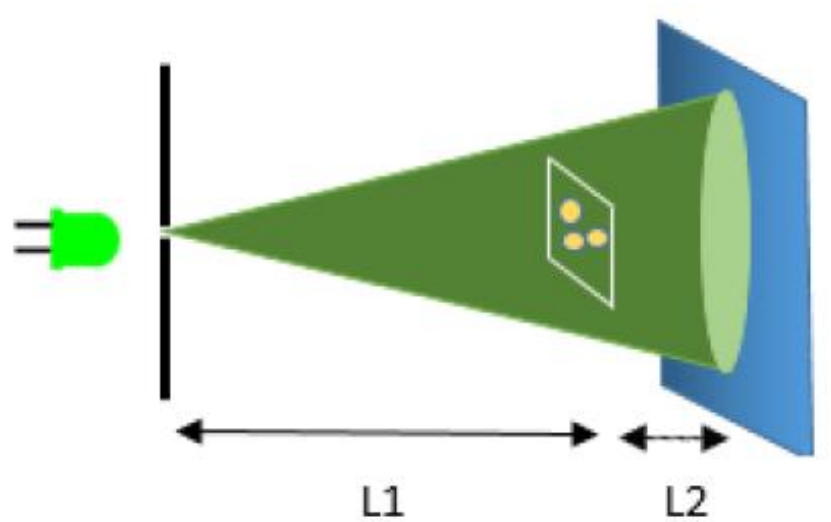

Fig -1: inline holographic setup

In general, the sample in inline holography is placed close to the pinhole and few centimeters away from the sensor [9]. The signature pattern of each cell will overlap with other at camera plane. This setup would also limit the field of view. Sample was placed close to the camera as shown in figure 1, where L1 is distance between pinhole and sample which is in the order of few centimeters; L2 is distance between sample and camera which is in order of few millimeters. This setup would increase the field of view of the sample.

\section{RECONSTRUCTION OF MICRO-OBJECT}

Inline hologram is recorded using CCD. As mentioned above optimization approach is used for reconstruction of object and detailed reconstruction procedure is given in [7]. The recorded hologram is given in equation below.

$$
H(x, y)=I(x, y)=\left|E_{0}\right|^{2}+\left|E_{R}\right|^{2}+E_{0} \cdot E_{R}{ }^{*}+E_{0}{ }^{*} \cdot E_{R}
$$

Where $E_{O}$ is the object beam and $E_{R}$ is reference beam. The unknown $\mathrm{E}_{\mathrm{O}}$ should satisfy

$$
H(x, y)-\left|E_{0}\right|^{2}+\left|E_{R}\right|^{2}+E_{0} \cdot E_{R}^{*}+E_{0}^{*} \cdot E_{R}=\text { Minimum. }
$$

Therefore, cost function is defined as shown in equation below

$$
\begin{aligned}
& \mathrm{F}\left(E_{0}, E_{0}^{*}\right)= \\
& \frac{1}{2}\left\|H(x, y)-\left|E_{O}\right|^{2}+\left|E_{R}\right|^{2}+E_{0} \cdot E_{R}{ }^{*}+E_{0}{ }^{*} \cdot E_{R}\right\|^{2}
\end{aligned}
$$

The optimization problem is solved by gradient descent scheme. The object is obtained by iterative calculation of equation given below

$$
E_{0}{ }^{n}=E_{0}{ }^{n-1}-\mathrm{t}[\nabla \mathrm{F}]_{E_{0}=E_{0}}{ }^{n-1}
$$

Where $E_{o}{ }^{n}$ is $n^{\text {th }}$ iterated value of object. Step size $t$ decides where our solution is heading towards which is calculated by line search method and $\nabla \mathrm{F}$ is gradient of cost function (gradient is taken with respect to $\mathrm{E}_{\mathrm{O}}{ }^{*}$ ) which is given in equation below

$$
\begin{aligned}
& \nabla \mathrm{F}= \\
& {\left[\left(H(x, y)-\left|E_{O}\right|^{2}+\left|E_{R}\right|^{2}+E_{0} \cdot E_{R}^{*}+E_{O}^{*} \cdot E_{R}\right) *\left(E_{O}+E_{R}\right)\right]}
\end{aligned}
$$

The iteration continues until $E_{o}{ }^{n}-E_{0}{ }^{n-1} \approx 0$. Thus the object which is obtained in this process is in hologram plane. Object field is then propagated from hologram plane to the image plane using Fresnel Kirchhoff integral [9] to obtain complex object. The distance between object and camera (hologram plane) is few $\mathrm{mm}$, therefore to calculate the exact distance of propagation autofocus algorithm [10] has been used. Since L1>>L2, each particle of sample is assumed to be illuminated by coherent source [8].

\section{RESULTS}

Ni-NTA (nickel nitrilotriacetic acid) agarose microbeads were used as micro-object. Beads should be sparsely distributed on a glass slide. The sample was placed closed to the sensor $(0-2 \mathrm{~mm})$. The recorded in line hologram is shown in the figure 2.

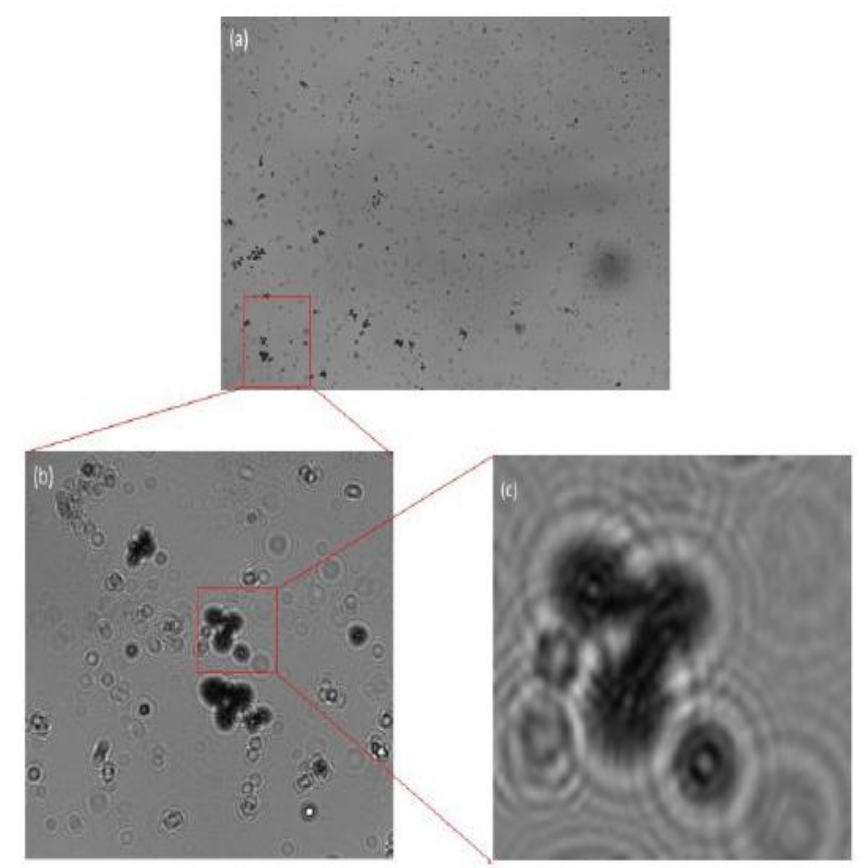

Fig-2: inline hologram

Field of view is more as it can be seen in figure 2(a). Diffraction pattern of beads is clearly visible as shown in the figure 2(c). The complex object field is reconstructed numerically. From the complex object field phase of the object is obtained as shown in figure 3 . 

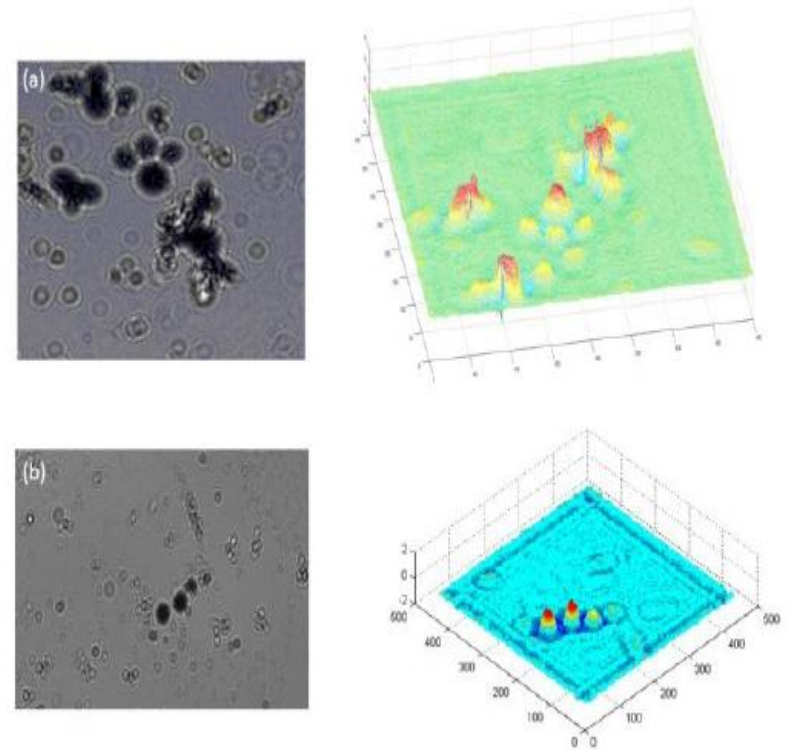

Fig-3: Reconstruction of microbeads, hologram(left), 3D reconstruction (right)

The reconstructed microbeads image is compared with the standard microscope as shown in figure 4.

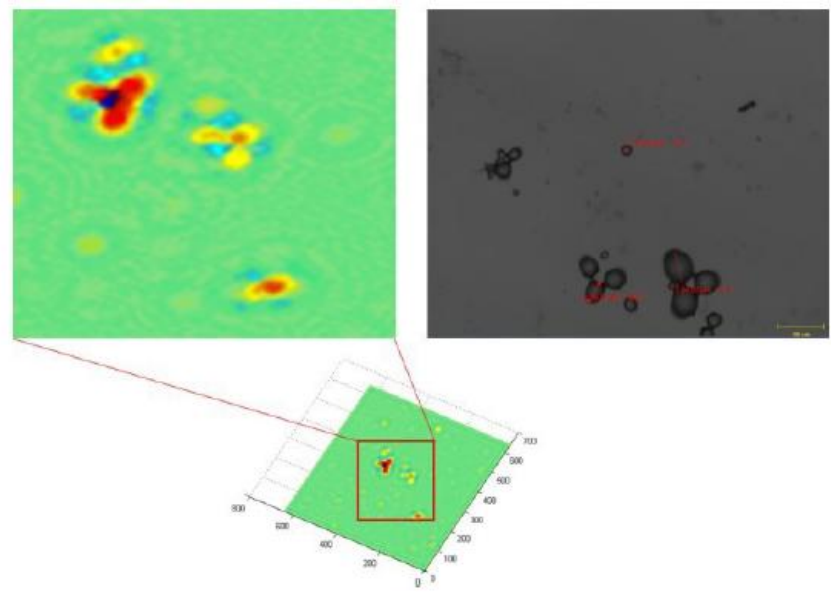

Fig-4: Comparison of low cost setup (left) and standard microscope (right) images

The low cost setup images can clearly show the microbeads. Reconstructed 3D images gave depth information as 3rd dimension which cannot be obtained from standard microscope. Thus, inline holography is promising tool for low cost implementation of 3-D microscopy.

\section{CONCLUSION}

Cost is main criteria in rural health care with accuracy in mind, inline holographic device can give good microscopic images. Initially micron size agarose beads were used for imaging, these can be extended to live cell imaging without need of any contrast agents. Phase information is also obtained, thus 3 rd dimension is achieved. This could also be extended to mobile platform by using phone camera instead of CCD sensor, further reducing the cost of the overall device.

\section{REFERENCES}

[1]. Gabor, D., A new microscopic principle. Nature, 1948. 161(4098): p. 777-778.

[2]. Leith, E.N. and J. Upatnieks, Reconstructed wavefronts and communication theory. JOSA, 1962. 52(10): p. 11231128.

[3]. Cuche, E., P. Marquet, and C. Depeursinge, Spatial filtering for zero-order and twin-image elimination in digital off-axis holography. Applied Optics, 2000. 39(23): p. 40704075.

[4]. Chen, G.-L., et al., Numerical suppression of zeroorder image in digital holography. Optics express, 2007. 15(14): p. 8851-8856.

[5]. Koren, G., F. Polack, and D. Joyeux, Iterative algorithms for twin-image elimination in in-line holography using finite-support constraints. Journal of the Optical Society of America A, 1993. 10(3): p. 423-433.

[6]. Latychevskaia, T. and H.-W. Fink, Solution to the twin image problem in holography. Physical review letters, 2007. 98(23): p. 233901.

[7]. Khare, K., P. Ali, and J. Joseph, Single shot high resolution digital holography. Optics express, 2013. 21(3): p. 2581-2591.

[8]. Mudanyali, O., et al., Compact, light-weight and costeffective microscope based on lensless incoherent holography for telemedicine applications. Lab on a Chip, 2010. 10(11): p. 1417-1428.

[9]. Schnars, U. and W. Jueptner, Digital holography2005: Springer.

[10]. Mudanyali, O., et al., Detection of waterborne parasites using field-portable and cost-effective lensfree microscopy. Lab on a chip, 2010. 10(18): p. 2419-2423. 